\title{
Quest for Band Renormalization and Self-Energy in Correlated $f$-Electron Systems
}

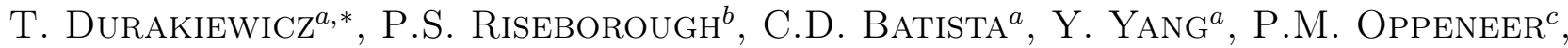 \\ J.J. JOYCE ${ }^{a}$, E.D. BAUER ${ }^{a}$ AND K.S. GRAHAM ${ }^{a}$ \\ ${ }^{a}$ Los Alamos National Laboratory, Condensed Matter and Thermal Physics Group \\ Mailstop K764, Los Alamos, NM 87545, USA \\ ${ }^{b}$ Department of Physics, Temple University - Philadelphia, PA 19122, USA \\ ${ }^{c}$ Department of Physics and Materials Science \\ Uppsala University - Box 530, S-75121, Uppsala, Sweden
}

\begin{abstract}
Coexisting low-energy scales are observed in $f$-electron materials. The information about some of low-energy scales is imprinted in the electron self-energy, which can be measured by angle-resolved photoemission (ARPES). Such measurements in $d$-electron materials over the last decade were based on high energy- and momentumresolution ARPES techniques used to extract the self-energy information from measured spectra. Simultaneously, many-body theoretical approaches have been developed to find a link between the self-energy and many-body interactions. Here we show the transcription of such methods from $d$-electrons to $f$-electrons by presenting the first example of low energy scales in the $f$-electron material $\mathrm{USb}_{2}$, measured with synchrotron-based ARPES. The proposed approach will help in answering the fundamental questions about the complex nature of the heavy fermion state.
\end{abstract}

PACS numbers: 79.60.-i, 71.28. + d, 74.25.Jb

\section{Introduction}

The electronic physics of a solid is governed by two basic principles: (i) the Pauli exclusion principle and (ii) the competition between interaction and kinetic energy terms. The kinetic energy stabilizes a "band-like" picture, in which uncorrelated electrons are delocalized over the lattice. On the other hand, the combination of electron-electron, spin-orbit and electron-phonon interactions introduce correlations that can produce strong deviations from this simple band-like picture. In some cases, these deviations correspond to the emergence of collective behaviour which may lead to band renormalization and formation of new states of matter. For instance, heavy fermion states, superconductors or Mott insulators emerge from collective responses of the many-body system. Such a coherent response of $10^{23}$ electrons establishes the physical properties of the material and contributes to the band renormalization. Details of the band renormalization mechanism, like coupling strength, characteristic energy scale and lifetime can be found in the self-energy $\Sigma=\operatorname{Re} \Sigma+\mathrm{i} \operatorname{Im} \Sigma$, a complex function that characterizes the interacting electron system. The self-energy is currently a very popular object of research

* corresponding author; e-mail: tomasz@lanl.gov in the $d$-electron high temperature superconductors, and it is usually extracted from a single crossing band exhibiting a "kink" in its dispersion [1,2]. An extraction of the self-energy allows direct measurement of the renormalization energy scale. The self-energy carries information about the coupling of electrons to bosonic modes, either phonons [3] or magnetic fluctuations [4], as well as electron-electron interactions, spin-orbit coupling and orbital hybridization. Hence, the self-energy treated as a measure of band renormalization is central in understanding and predicting of a correlated system behaviour.

For about a decade, the self-energy language has been used to describe the nature of many body interactions in high temperature superconductors. A great body of work has been devoted to the extraction of the self-energy from kinks observed in the band dispersion measured with angle resolved photoemission (ARPES) having a high energy and momentum resolution. The effort of transcribing the experimental tools developed for high temperature superconductors onto the area of $f$-electrons was initiated at Los Alamos National Laboratory (LANL) most recently $[5,6]$. This initial work reveals that a finite value of the real part of self-energy exists and can be measured in $f$-electron systems. This non-zero self-energy comes from many-body interactions related to the emergence of a coherent, heavy electron state. 


\section{Methods and results}

Several flux-grown single crystals of $\mathrm{USb}_{2}$ were cleaved in the ultra-high vacuum environment of less than $3 \times$ $10^{-11}$ Torr. A Neel temperature of $205 \mathrm{~K}$ was found from magnetic susceptibility measurements, and all ARPES measurements were performed at $15 \mathrm{~K}$, well inside the antiferromagnetic phase. Sample orientation was determined by X-ray diffraction (Laue camera). Angle-resolved photoemission measurements were performed using the Plane Grating Monochromator beamline at the Synchrotron Radiation Center in Stoughton, Wisconsin. A photon energy of $34 \mathrm{eV}$ was used, with measurement energy resolution better than $20 \mathrm{meV}$ and an acceptance angle of \pm 1 degree. The Fermi level position of the sample was referenced to the Fermi level of Pt. The components of the quasiparticle peak, its binding energy and width were established by a nonlinear multiparameter regression fitting of the experimental data for each $\boldsymbol{k}$ value.

The electronic structure of $\mathrm{USb}_{2}$ along the $\Gamma$ to $X$ direction is shown in Fig. 1, where the photoemission intensity is normalized only by the photon beam intensity. The bright band marked as A in both panels of Fig. 1 is the quasiparticle band being discussed here. The top panel in Fig. 1 shows the energy distribution curve (EDC) in the vicinity of the $\Gamma$ point, with a scale expanded to show the $\mathrm{B}$ and $\mathrm{C}$ bands clearly. The line represents smoothed data as guide to the eye. The bands A, B and $\mathrm{C}$ are marked with arrows. The contour plot of the EDCs between $\Gamma$ and $\mathrm{X}$ points is shown in the lower panel in Fig. 1, and the characteristic features A, B and C are marked the same as in the top panel.

The LSDA calculations were performed within the full potential local orbital method [7] with the local spin-density approximation (LSDA). The calculated LSDA band structure is also shown in black lines in the lower panel of Fig. 1. The $f$-electrons were treated as itinerant and included in the set of valence states in the fully-relativistic and full-potential calculations using the antiferromagnetic superstructure of $\mathrm{USb}_{2}$. The PerdewWang parameterization [8] of the LSDA exchange and the correlation functional was also used.

On a large energy scale, good agreement is obtained between the energies of the peaks $\mathrm{A}, \mathrm{B}$ and $\mathrm{C}$ with the LSDA band energies at the high-symmetry point $(\Gamma)$. However, as $k_{x}$ is increased towards $X$ point, one can clearly notice discrepancies in the vicinity of the Fermi level. In addition to being shifted away from the Fermi energy, the measured band dispersion is flatter than in LSDA calculations, as shown in Fig. 2. The flattening of the band is restricted to energies within the first 20-50 meV below the Fermi level.

\section{Discussion}

The zero-area Fermi surface renormalization model, illustrated in Fig. 3 was proposed $[5,6]$ to explain the experimental findings in agreement with the results of
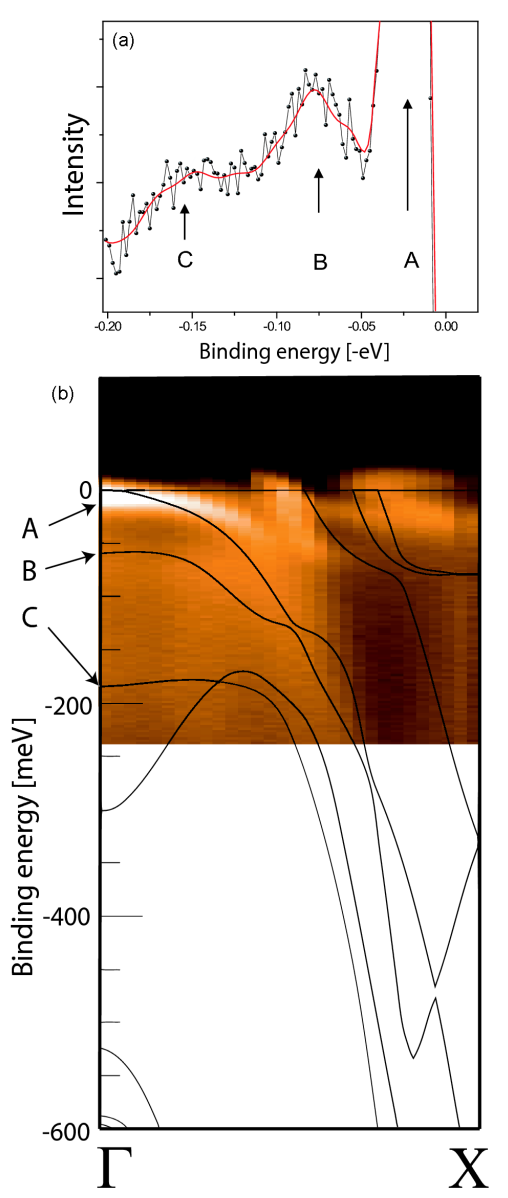

Fig. 1. Results of ARPES measurements of the electronic structure of $\mathrm{USb}_{2}$. Measurement is performed along the $\Gamma$ to $X$ direction in the Brillouin zone. The energy distribution curve is shown in panel (a), where three characteristic features $\mathrm{A}, \mathrm{B}$, and $\mathrm{C}$ are indicated with arrows. The same three features are marked in panel (b), where the experimental data are shown on top of our LSDA band structure calculation. Discrepancies between the LSDA calculation and experiment are seen in the intensive band A near the Fermi level, and are caused by band renormalization due to many-body effects, which are not present in the LSDA calculation.

the many-body calculations, which revealed a quadratically dispersing, narrow and coherent quasiparticle width for small values of momentum. The effective mass for small $\boldsymbol{k}$ is twice larger than seen for larger values of $\boldsymbol{k}$, and the crossover region between two different dispersion rates is formed, manifesting itself as a kink (Fig. 2). The band renormalization and corresponding formation of kink energy scale and gap energy scale are linked to the interband scattering process, and not to the standard electron-phonon mass enhancement. The self-energy reflects the small energy scales created through low-energy transitions from the osculating band into a band that does cross the Fermi energy. As shown in Fig. 4, because of the electron-electron interaction through interband scattering, a narrow gap is produced in the imagi- 


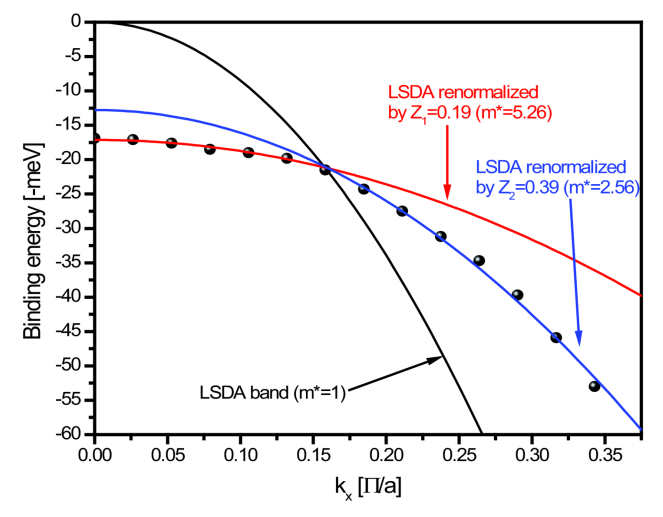

Fig. 2. Kink in the band dispersion in $\mathrm{USb}_{2}$. The kink in the band dispersion appears near $E_{\mathrm{B}}=-20 \mathrm{meV}$ and $k_{x}=0.18$, and marks the boundary between two distinct renormalization areas.

nary part of self-energy, with the imaginary part falling rapidly to zero inside the gap. This leads to a variation of the real part of self energy, and, consequently, results in the quasiparticle mass enhancement.

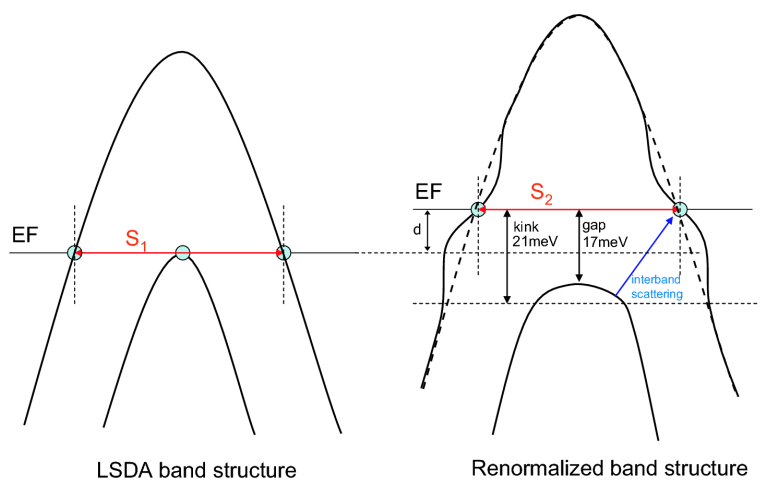

Fig. 3. Small energy scales in $\mathrm{USb}_{2}$. The renormalization of the point-like Fermi surface leads to flattening of the osculating band and to the shift in the Fermi energy. Due to renormalization, the kink scale of $21 \mathrm{meV}$ emerges together with the gap scale of about $17 \mathrm{meV} . S_{1}$ and $S_{2}$ represent the Fermi volume before and after renormalization, where the Luttinger's condition $S_{1}=S_{2}$ is maintained.

\section{Future directions}

The example of $\mathrm{USb}_{2}$ shows that measurement of the self-energy is possible in $f$-electron systems. The extraction of inherent low-energy scales, if succesful, will help our understanding of the more archetypal heavy fermion systems like $\mathrm{UPd}_{2} \mathrm{Al}_{3}$ or members of the CeMIn 5 family, where $\mathrm{M}=\mathrm{Co}$, Ir or Rh. It is widely accepted that the coherent behaviour emerges in heavy fermions at an unified temperature scale, $T^{*}$, manifested by several thermodynamic properties of heavy electron materials [9]. Numerous unusual physical properties appear in heavy fermion

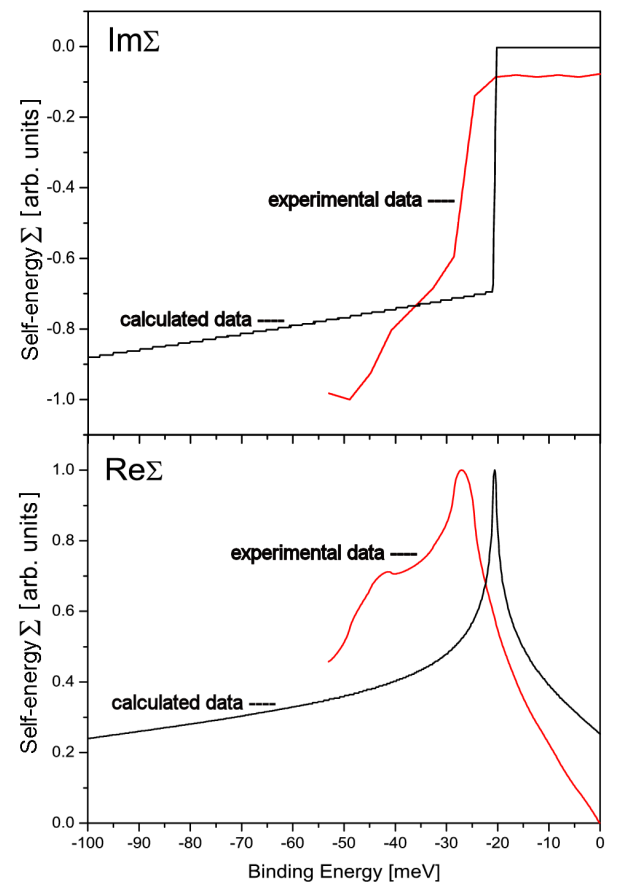

Fig. 4. Electron self-energy in $\mathrm{USb}_{2}$. In the figure the real and imaginary parts of the self-energy extracted from experimental data and calculated ones are shown. The real part of the self-energy function was found self-consistently by a Kramers-Kroning transformation of a fitted spectrum. Note the zero value of the imaginary part inside the gap, as well as a large variation of the real part at the kink energy scale.

materials when temperature is lowered and many-body effects lead to band renormalization. Local $f$-electron moments may be treated as atoms at very high temperatures, while inter-atomic coherence takes place below the temperature $T^{*}[10]$. Below $T^{*}$, electrons start propagating coherently (in contrast to the diffusive motion above $T^{*}$ ) leading to a Fermi liquid with heavy quasiparticles. The crossover between the two regimes is reflected in the thermodynamic properties. The magnetic susceptibility ceases to be Curie-Weiss like below $T^{*}$ and the magnetic entropy starts to be quenched [11]. This is accompanied by a drop in resistivity, and an increase in the spectral weight at the Fermi level. A "dual nature" behaviour is therefore seen, where localized and itinerant $f$-electrons coexist. Spectroscopically, $T^{*}$ scale is relatively large and may be associated with evolution of the hybridization gap. The first attempts to extract the hybridization gap information through temperature-dependent ARPES measurement are ongoing.

Below $T^{*}$ the electronic structure of heavy fermion materials is governed by renormalization of electron mass due to correlation effects coming from many-body interactions [12]. A fundamental question is to identify the responsible interaction or, correspondingly, the microscopic origin of the dominant energy scale $T^{*}$. Although recent experimental analysis suggests that $T^{*}$ is given by the 
coupling between neighboring f-moments through conduction electrons (Ruderman-Kittel-Kasuya-Yosida or RKKY coupling), it is conventionally attributed to the "so-called" lattice Kondo effect involving crystal field excitations [11]. Distinguishing these two scenarios is one of the central issue of the heavy electron research. Their delicate difference may be reflected in the self-energy. Moreover, while $T^{*}$ marks the onset of the heavy electron coherence, heavy fermion materials have yet another lower temperature scale $\left(T_{\mathrm{FL}}\right)$ below which the heavy electron coherence is fully developed and a Fermi liquid state is established. All these can be studied by the temperature evolution and relevant features of the self-energy obtained from future ARPES measurements

\section{Conclusions}

Building on our previous experience with $\mathrm{USb}_{2}$ [13], in 2008 we have discovered a small energy scale in this compound via the observation of a kink in $f$-electron dispersion [5, 6], see Fig. 1, panel B. The kink structure was observed for the first time in any $f$-electron system. The kink energy scale of $21 \mathrm{meV}$ and the ultra-small intrinsic peak width of $3 \mathrm{meV}$ were observed. This finding extended the context of quasiparticle band renormalization from $d$ to $f$-electrons, hence creating a link between high temperature superconductors and heavy fermions and actinide based compounds. A new model of point-like Fermi surface renormalization was proposed to explain the spectroscopic properties of the kink. Work on identification of numerous small energy scales in heavy fermion systems $\mathrm{CeIrIn}_{5}$ and $\mathrm{UPd}_{2} \mathrm{Al}_{3}$ is ongoing.

With the first measurements described here, the quest towards finding the low energy scales in $f$-electron materials by utilizing self-energy extraction from AREPS measurements has started. Except for the most recent effort at LANL, there is yet no experimental information about spectral properties of coherent quasiparticles in $f$-electron systems in terms of electron self-energy. Only limited data of resolution-limited quality [14] are available for the temperature evolution of the quasiparticle weight through $T^{*}$. This void will be filled in the years to come by utilizing the high resolution, temperature-dependent ARPES techniques.

\section{Acknowledgments}

Work supported by the US Department of Energy, Office of Science, Division of Materials Science and Engineering, and the LANL LDRD Program. The SRC is operated under Grant No. DMR-0537588. The work at Temple was funded by DOE Office of Basice Energy Science, Materials Science through DEFG02-01ER45872.

\section{References}

[1] S. Hufner, Very High Resolution Photoelectron Spectroscopy, Springer 2007

[2] A. Damascelli, et al., Rev. Mod. Phys. 75, 473 (2003).

[3] A. Lanzara, et al., Nature 412, 510 (2001).

[4] P. D. Johnson, et al., Phys. Rev. Lett. 87, 177077 (2001).

[5] T. Durakiewicz, et al., Europhys. Lett. 84, 37003 (2008).

[6] X. Yang, et al., Phil. Mag. 89, 1893 (2009).

[7] K. Koepernik, H. Eschrig, Phys. Rev. B 59, 1743 (1999).

[8] J.P. Perdew, Y. Wang, Phys. Rev. B 45, 13244 (2004).

[9] Yi-feng Yang et al., Nature 454, 611 (2008).

[10] J.H. Shim, et al., Science 317, 1615 (2007).

[11] S. Nakatsuji, et al., Phys. Rev. Lett. 92, 016401 (2004).

[12] F. Steglich, et al., Phys. Rev. Lett. 43, 1892 (1979).

[13] E. Guziewicz, et al., Phys. Rev. B 69, 45102 (2004).

[14] S.-I. Fujimori, et al., Nature Physics 3, 618 (2007). 